\title{
Cathodoluminescence and DART Mass Spectrometry for the Forensic Identification of Explosive and Narcotic Particle Residues on Surfaces
}

\author{
Scott Wight and Greg Gillen
}

National Institute of Standards and Technology, Materials Measurement Science Division, Gaithersburg, Maryland, USA.

The Materials Measurement Science Division at NIST has a significant effort in support of trace contraband detection and forensic microscopy and analysis. Characterization of the particle characteristics (size, morphology, chemical composition and areal coverage) of trace contraband residues on surfaces is critical for developing optimized sampling strategies for current and emerging trace detection technologies. Furthermore, a detailed understanding of the nature of these residues is important for the development of appropriate and realistic test materials. At NIST we are employing a variety of analytical tools to characterize such residues on surfaces. These tools include polarized light microscopy (PLM), Raman, Secondary Ion Mass Spectrometry (SIMS), Scanning Electron Microscopy (SEM) and ambient mass spectrometry. In this work we present the first (to our knowledge) evaluation of Cathodoluminescence Microscopy (CL) for the chemical identification and imaging of a variety of particle residues from both explosives and illicit narcotics. Previous work in the Cathodoluminescence of pharmaceutical materials demonstrated a surprisingly large number of CL active materials [1]. The samples were examined in an FEI Quanta 200F environmental SEM fitted with a Gatan MonoCL4 Cathodoluminescence system [2]. Samples were typically dispersed as pure powders or mixtures of powders on gunshot residue stubs (GSR mounts) and examined without further preparation. Samples included a range of illicit narcotics, cutting agents, common explosives and homemade explosive precursors. Prior to analysis by CL, each of the samples was characterized by Direct Analysis Real Time Mass Spectrometry (DART MS) to confirm the chemical identity of the test particle residues and to evaluate the purity of the samples. Representative CL spectra and images as well as secondary electron images were obtained from each of the materials using several different primary electron beam energies. CL images of micrometer sized particles typically show more uniform illumination and less sensitivity to sample topography and sample charging compared to conventional SEM imaging. This can greatly simplify automated particle recognition, sizing and counting of contraband particles in the SEM. In comparison to the other techniques listed above, CL microscopy allows chemical mapping of contraband particles at SEM spatial resolution which is particularly useful for characterization of micrometer to sub micrometer diameter particles. Examination of simulated fingerprint depositions, where the particles were buried in sebaceous secretions demonstrated the CL imaging was still feasible and provided more robust localization of contraband particles compared to conventional SEM. We will also demonstrate the feasibility of using CL to distinguish between different explosive polymorphs. Overall, CL imaging appears to provide a useful new tool to further understand the characteristics of trace contraband residue particles, sample results from Methamphetamine (Figure 1) and TNT (Figure 2) are included below.

\section{References:}

[1]G Nichols, European Journal of Pharmaceutical Sciences, 45 (2012) p.19.

[2] Certain commercial equipment, instruments, or materials are identified in this report to specify adequately the experimental procedure. Such identification does not imply recommendation or endorsement by the National Institute of Standards and Technology, nor does it imply that the materials or equipment identified are necessarily the best available for the purpose. 


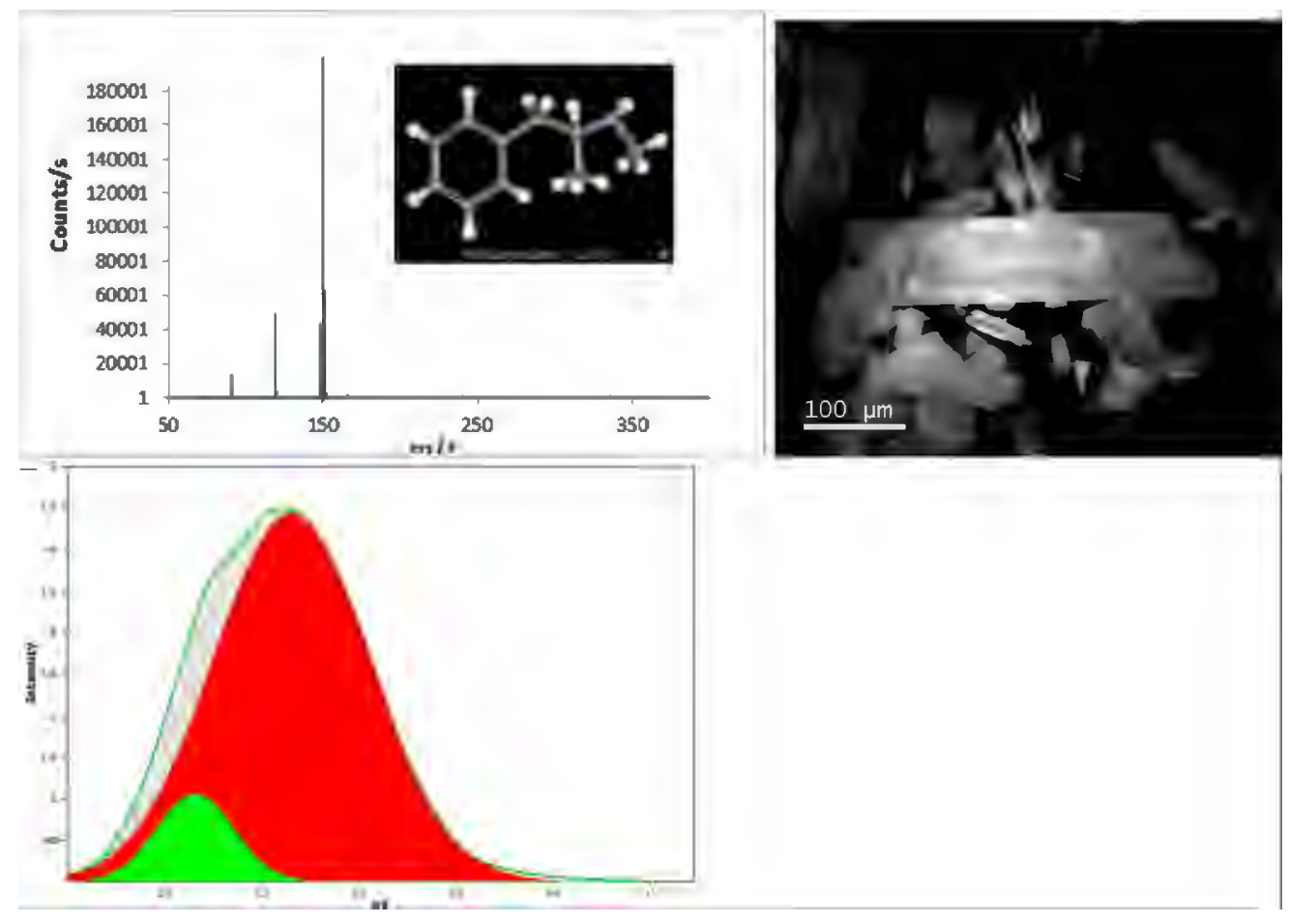

Figure 1. Methamphetamine: DART spectrum with inset model, Panchromatic CL image, and CL spectrum fit with two Gaussians at $2.16 \mathrm{eV}$ and $2.66 \mathrm{eV}$.
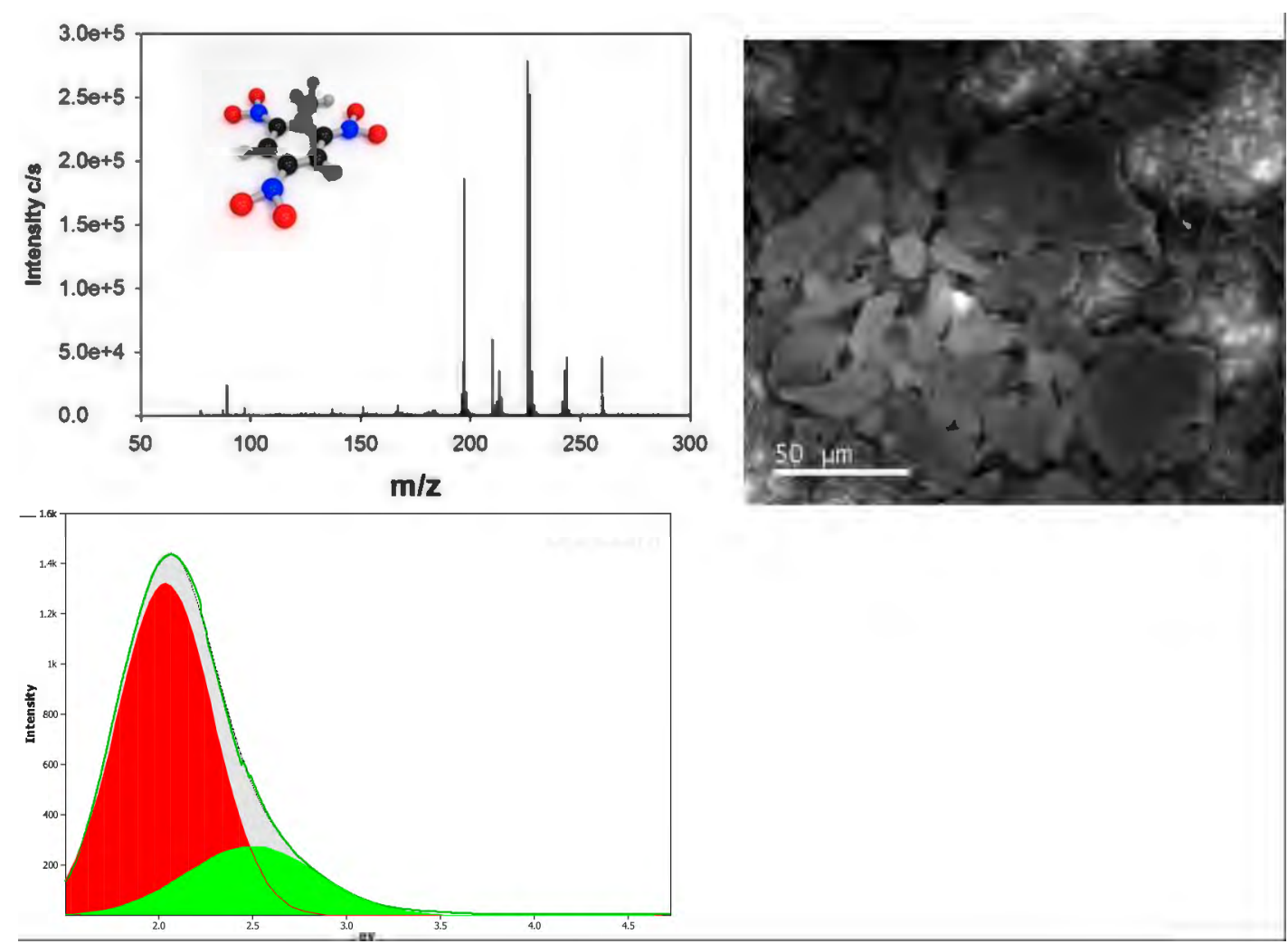

Figure 2. TNT: DART spectrum with inset model, Panchromatic CL image, and CL spectrum fit with two Gaussians at $2.04 \mathrm{eV}$ and $2.5 \mathrm{eV}$. 